\title{
EL ENFOQUE DE LA CARTOGRAFÍA SOCIAL PARA EL ANÁLISIS DE DEBATES SOBRE POLITICAS EDUCATIVAS
}

\section{THE CONTRIBUTIONS OF SOCIAL CARTOGRAPHY APPROACH TO EDUCATION POLICY ANALYSIS}

\author{
César Tello* \\ Jorge M. Gorostiaga **
}

\begin{abstract}
Resumo
O enfoque da Cartografia Social para a análise de debates sobre políticas educacionais

Este artigo descreve as principais características do enfoque da cartografia social para o estudo e análise das políticas educacionais. Uma das questões centrais da cartografia social é que ela permite visualizar - e eventualmente integrar - diferentes perspectivas que conceitualizam o fenômeno e verificar como essas perspectivas se integram ou se inter-relacionam. A cartografia social como enfoque tem seu potencial para a análise das políticas educacionais na busca de implicações da multiplicidade de discursos ou perspectivas discursivas, assim como sua inter-relação, constituindo-se como uma ferramenta de análise sumamente valiosa que pode ser combinada com outros métodos e técnicas para o estudo crítico de diversos problemas educacionais.
\end{abstract}

Palavras-chave: Cartografia social. Pesquisa. Políticas educacionais.

\section{Resumen}

Este artículo describe las principales características del enfoque de la cartografía social para el estudio y análisis de las políticas educativas. Una de las cuestiones centrales de la cartografía social es que permite visualizar - y eventualmente integrar - diferentes perspectivas que conceptualizan el fenómeno, y cómo estas interactúan o se interrelacionan. La cartografía social como enfoque tiene su potencia para el análisis de las políticas educativas en la búsqueda de las implicancias de la multiplicidad de discursos o perspectivas discursivas, así como su interrelación, constituyéndose como una herramienta de análisis sumamente valiosa que puede ser combinada con otros métodos y técnicas para el estudio crítico de diversos problemas educativos.

Palabras Claves: Cartografía social. Investigación. Políticas Educativas.

\begin{abstract}
This article describes the main features of social cartography to the study and analysis of educational policies. One of the main advantages of social cartography is that it allows the visualization - and eventually the integration of different perspectives that conceptualize the phenomenon being investigated and verifies these perspectives as they integrate and relate to one another. Social cartography has the potential for the analysis of educational policies in search of the implications of the various discourse or discursive perspectives as well as their inter-relation. Therefore, social cartography becomes a valuable tool for analysis that can be combined with other methods and techniques for investigating numerous educational problems.
\end{abstract}

Keywords: Social cartography. Research. Educational policies.

\section{Presentación}

Este artículo aborda y desarrolla el enfoque de la cartografía social a través de tres estudios de aplicación en investigación para el análisis de políticas educativas. Nuestro objetivo es mostrar el valor heurístico y de creación de conocimiento de este enfoque en un contexto de creciente diversidad de visiones sobre aspectos teóricos, de política y de la práctica en la educación (PAULSTON, 2001; GOROSTIAGA, en prensa).

La cartografía social ${ }^{1}$ es un enfoque metodológico basado en el análisis textual que busca la representación de fenómenos sociales a través de mapas que reinscriben y estructuran una multiplicidad de perspectivas o formas de ver estos fenómenos. Su mayor desarrollo se ha dado dentro del campo de la educación comparada a partir de los trabajos que

\footnotetext{
${ }^{1}$ Según hemos podido relevar, existen otros usos y aplicaciones de la Cartografía Social que, aunque comparten la concepción medular de representación visual, poseen como eje, en el caso de la sociología, la Investigación Acción Participante (ANDRADE, 1997; HABEGGER; MANCILA, 2006). En el campo de la geografía se la utiliza para referirse a enfoques que toman en cuenta los contextos socio-culturales de producción y "morfología humana" a través del uso de de mapas (BUZAI, 2003; CLAVAL, 1999).

"Profesor en la Universidad Nacional de San Martín, Universidad Nacional de La Plata e Universidad Nacional Tres de Febrero (Argentina). E-mail: cesargeronimotello@yahoo.com.ar

" Profesor en la Universidad Nacional de San Martín. Investigador de lo Consejo Nacional de Investigaciones Científicas y Técnicas CONICET (Argentina). E-mail: jgorosti@unsam.edu.ar
} 
llevó a cabo el académico norteamericano Rolland Paulston en la Universidad de Pittsburgh desde principios de la década de 1990.

La descripción y el análisis que se presenta en este trabajo sobre la cartografía social (CS) posee su base empírica en tres estudios (GOROSTIAGA, 2003; GOROSTIAGA, TELLO, MARTINÉ, 2008; TELLO, 2009) que hemos llevado a cabo hasta el momento partiendo de este enfoque. En este artículo explicaremos los supuestos del enfoque de la CS y la metodología propiamente dicha: el mapeo de discursos, para ejemplificar con los estudios mencionados y explicar las particularidades que cada uno de ellos ha tomado de acuerdo a las características propias de cada investigación.

De esta forma podremos pensar en las potencialidades que la CS posee para el análisis de políticas educativas en Latinoamérica, como así también otras posibles aplicaciones en la investigación educativa en nuestra región.

\section{La Cartografía Social como enfoque}

Consideramos la cartografía social como enfoque, dado que asume una visión sobre la realidad en términos epistemológicos y propone una metodología específica -la combinación de análisis textual y mapeo- para desarrollarla. La CS asume una mirada poscrítica que se combina con elementos del pensamiento complejo de Edgar Morin.

La perspectiva poscrítica, siguiendo el análisis de Silva (2001), daría cuenta de una difusa frontera respecto de las teorías críticas, pero asumiendo una perspectiva posmoderna. Conociendo la crítica que recibe la posmodernidad como espacio de la fragmentación, como mirada superflua o escéptica, y particularmente en educación y desde la perspectiva académica (ver, por ejemplo, FOLLARI, 2002), es que se hace necesario explicar algunos de los pilares que sostienen la perspectiva poscrítica como parte del enfoque posmoderno, en tanto la comprendemos como un modo epistemológico de abordar una realidad cada vez más cambiante y discontinua. A partir del análisis de Paulston (2001) destacamos como características de la perspectiva poscrítica entendida en el marco del enfoque posmoderno:

a) el rechazo a los fundamentos del iluminismo, como conocimiento acabado y "verdadero";

b) rechazo al eurocentrismo y las prácticas poscoloniales;

c) rechazo a las posiciones binarias como formas de conocimiento;

d) Se asume un viraje en el modo de investigar: del tiempo al espacio, de los hechos a las interpretaciones, de posiciones firmemente asentadas a la lectura de narrativas, y de verificación de proposiciones a la representación de diferencias.

En este sentido, la CS postula que deben abandonarse los actuales sistemas conceptuales basados en nociones como centro, margen, jerarquía y linealidad y sustituirlos por otras de multilinealidad, nodos, nexos y redes. El enfoque constituye un cuestionamientofundamentalalalógicasubordinativa que ha imperado en el pensamiento occidental, y una reivindicación de los modelos asociativos y coordinativos que se encuentran en concordancia con el paradigma holístico y trans-disciplinario de la postmodernidad (VÁSQUEZ ROCCA, 2001).

La perspectiva poscrítica en la que situamos a la CS reconoce un lugar protagónico otorgado a lo discursivo, la presencia de la contingencia, de relaciones discursivas, lo diferente, antagónico, los lugares y los no-lugares en un debate sobre políticas educativas. El enfoque de la CS asume como posición epistemológica, en el sentido de Peters (2001), rehusarse a abordar el conocimiento sobre la realidad como una representación precisa de la misma.

En esta línea de análisis, Rolland Paulston (2001) entiende el enfoque de la CS como una nueva percepción para acrecentar la capacidad de reflexión, concebir la multiplicidad y "contrastarla con la armonía y la racionalidad apolínea” (p. 19). Refiriéndose al valor de los mapas, Paulston (2001) explica que:

Esta representación espacial o mapa de posiciones y relaciones de saber, representación enredada e interconectada (o rizoma deleuziano), puede ser visto como una metáfora del debate, como un enfoque heurístico y como un sitio real de paralogía y proceso posmoderno. También puede ser visto como una nueva y útil herramienta específicamente creada para dar forma visual a la creciente complejidad del trabajo del conocimiento en la actualidad (p. 19).

En el sentido planteado por Paulston (1995, 2001), la CS comparte, desde nuestra perspectiva, algunos elementos del pensamiento complejo de Edgar Morin, en tanto asume la categoría de complexus como aquello que "está tejido junto". El pensamiento complejo es un pensamiento que trata a la vez de vincular y de distinguir, pero sin desunir. El dogma de un determinismo universal se ha derrumbado (MORIN, 1999). El universo no está sometido a la soberanía absoluta del orden, sino que es el campo de acción de una relación dialogal-discursiva entre el orden, el desorden y la organización.

El pensamiento complejo es, esencialmente, el pensamiento que integra la incertidumbre y es 
capaz de concebir la organización. Que es capaz de reunir, contextualizar, globalizar, pero reconociendo lo singular y lo concreto. En esto consiste la epistemología de la cartografía social. Como afirma Wallerstein (1997), "es posible que estemos presenciando el fin de un tipo de racionalidad que ya no es apropiada para nuestro tiempo. Pedimos que se ponga el acento en lo complejo, lo temporal y lo inestable". (p. 8).

La CS consiste en desarrollar un enfoque que permita "identificar y comparar la diferencia" (PAULSTON; 1996, p. 19). Un elemento fundamental de la CS es el enfoque fenomenográfico, definido como "el estudio empírico de las diferentes y limitadas formas cualitativas en las cuales varios fenómenos y aspectos del mundo a nuestro alrededor son experimentadas, conceptualizadas, entendidas, percibidas y aprehendidas" (MARTON, 1994, p. 4424, nuestra traducción). Para un determinado tema o campo teórico, este enfoque permite identificar y caracterizar los argumentos (y presuntos saberes) de diversas perspectivas, y analizar las relaciones entre estas perspectivas. Esto resulta particularmente relevante en un escenario de creciente relativismo y fragmentación (LIEBMAN; PAULSTON, 1994) a la vez que justifica la representación de una multiplicidad de visiones en lucha - multiplicidad que en las ciencias sociales ya había sido advertida por Giddens (1979) - afín a la sensibilidad posmoderna. En este sentido, Paulston (2001) plantea que:

Quizás la característica más importante de la sensibilidad posmoderna sea un viraje ontológico desde una visión esencialista de una realidad fija, en la cual la razón es el principio que controla el universo, hacia una visión antiesencialista en donde las construcciones de la realidad aparecen resistiendo su clausura, y donde múltiples y diversos postulados de verdad se vuelven parte de una lucha constante (p. 19).

La cartografía social propondría una interpretación de cómo distintos discursos interactúan y cómo visualizan determinada cuestión educativa. En este artículo se mostrarán tres ejemplos de aplicación de la CS a distintos temas de políticas educativas concebidos como debates intertextuales: la descentralización escolar (GOROSTIAGA, 2003); la relación entre reformas educativas y globalización en América Latina (GOROSTIAGA, TELLO, MARTINÉ, 2008); y las políticas docentes en Latinoamérica (TELLO, 2009).

\footnotetext{
${ }^{2}$ Gorostiaga (en prensa) argumenta que la CS ha sido empleada con tres usos principales: el mapeo de campos teóricos; el mapeo de debates de política educativa; y la evaluación de programas o políticas.
}

\section{El Mapeo como metodología de la Cartografía social}

La metodología del mapeo implica una serie de procedimientos que hagan posible la identificación/ construcción de las diversas visiones, a las que denominamos Perspectivas o Comunidades en un determinado campo del debate. Esto se realiza llevando a cabo una lectura profunda y minuciosa (close reading) o exégesis de cada texto para develar su cosmovisión, sus posiciones de pensamiento, postulados de verdad o argumentos, sus estrategias de argumentación, etc, con el objeto de identificar las principales visiones y por ende agrupar los textos dentro de las diversas perspectivas que conformarán las comunidades. La categoría de Comunidad es utilizada según las investigaciones que presentamos para agrupar diversos textos que poseen una misma visión sobre el objeto de estudio. Así se puede encontrar lo que Gorostiaga (en prensa) denomina "comunidades de saber" para hacer referencia a corrientes de pensamiento o escuelas teóricas. En ambos casos, pueden verse como variantes (sub-discursos) del discurso sobre un determinado problema o área de la política o la práctica, o sobre un determinado campo del conocimiento. Y con otro uso metodológico lo que Tello (2009) denomina "comunidades discursivas" como el agrupamiento de perspectivas con una visión compartida de significados (véase Figura $V$ ). Esta última se puede graficar esquemáticamente del siguiente modo:

El análisis de textos constituye la principal herramienta para la identificación de perspectivas o formas de ver un determinado fenómeno. En la mayoría de los casos, se trata de una cantidad considerable de textos, lo cual implica favorecer la identificación de los principales supuestos, argumentos y características retóricas salientes de cada texto en relación al universo de textos elegidos, antes que un estudio detallado de los aspectos lingüísticos de cada texto. Un punto importante a tener en cuenta es que el propósito de la CS es el de leer e interpretar los textos, no los autores. Esto requiere que, en la medida de lo posible, se le permita a los textos hablar por sí mismos; contar, con el uso de citas, sus propias historias. El uso de citas en la presentación de los resultados de la investigación ayuda también a mostrar que la interpretación del texto por parte del investigador no es arbitraria, a la vez que deja abierta la posibilidad de nuevas interpretaciones por parte del lector (GOROSTIAGA, en prensa).

Como estrategia poscrítica, la CS hace visible la hipertextualidad de sentidos que comparten diversas perspectivas y comunidades en la propia interacción cartográfica evitando una mirada de totalidad, sino de superposición y yuxtaposición 


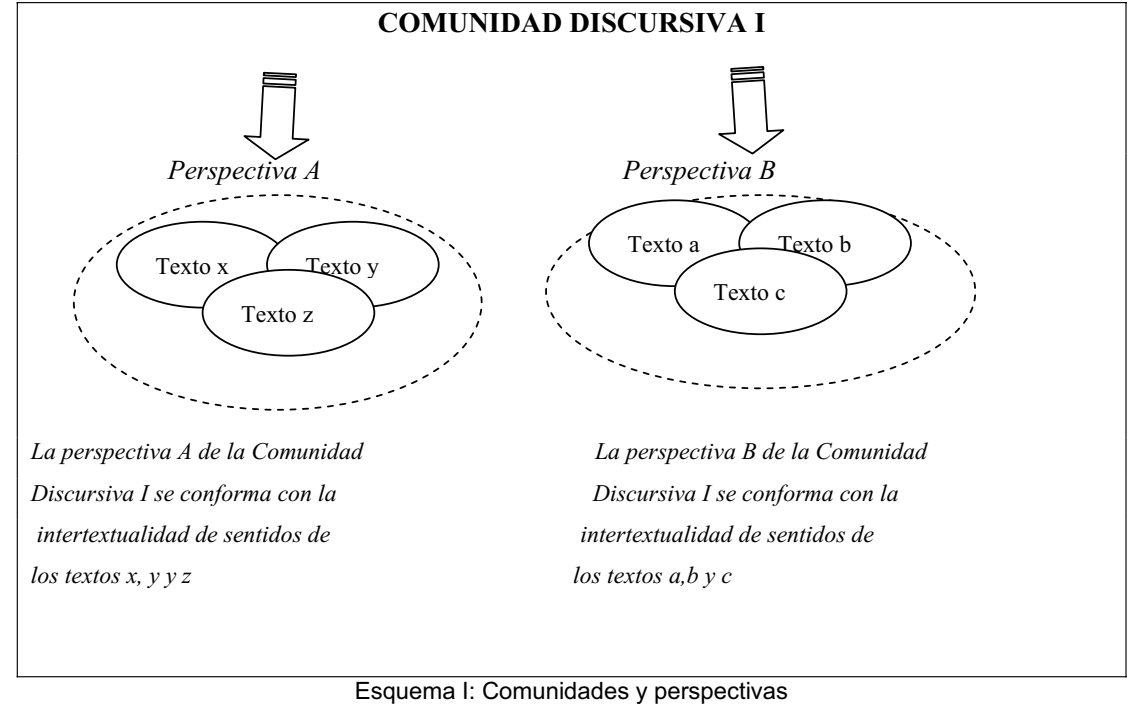

de significados sin sellar fronteras en el discurso. Esto produce un dinamismo cartográfico que está dado por lo que Paulston (2001) denomina flujos discursivos, en tanto se da una interacción entre las diversas perspectivas

La concepción de flujo discursivo nos permite reconocer que en el dominio de lo simbólico el mapa encierra una extrema dinámica, pues está basada en relaciones en flujos discursivos y por lo tanto posee efectos de sentido. Esto se puede representar a través de flechas ${ }^{3}$ para indicar la genealogía y las direcciones de las relaciones de conocimiento entre los distintos elementos del mapa. Como se observa en la Figura 1 (veáse también Figuras 3, 4 y 5)

De esta forma el mapa se caracteriza por la "heterotopía" en el sentido de Foucault (1986). En contraste con el espacio Utópico, las heterotopias se caracterizan por "ser capaces de yuxtaponer en un único espacio real varios espacios, varios sitios que son en sí mismo incompatibles" (FOUCAULT, 1986, p. 25).

Adicionalmente, Ruitenberg (2007) argumenta que los mapas son una forma alternativa o complementaria de discurso que puede ayudar a examinar el carácter espacial de las experiencias educativas y que es más adecuado que el discurso narrativo para capturar y eventualmente cuestionar las interconexiones de las prácticas hipertextuales de lectura y escritura cada vez más presentes en el campo educativo.

Por otra parte, Ninnes y Mehta (2004) subrayan en referencia al mapa llevado a cabo por Paulston (2001) sobre posmodernidad y educación comparada (Figura 1), pero aplicable a los estudios presentados en este trabajo, que estos mapas constituyen un viraje

\footnotetext{
${ }^{3}$ Las flechas de línea continua indican cómo una perspectiva se apoya en otra/s para desplegar sus argumentaciones, las flechas de línea discontinua indican la oposición argumental en su cosmovisión.
}

radical en la conceptualización del campo de la educación comparada en tanto representan una construcción ecléctica, antihegemónica, múltiple, porosa y dinámica (en contraste con la visión prevaleciente de un campo fijo, unificado, coherente e impermeable) y un punto de partida para la continuación del diálogo (en vez de una respuesta definitiva).

Para el análisis textual se puede utilizar el esquema desarrollado por Gorostiaga (2007): 1. Estructura: a) Tipo de texto: documento de política, ensayo académico, informe científico, etc. b) Organización del texto: principales secciones y funciones 2. Características retóricas (el modo en que se argumenta y el "tono" discursivo.) a) Lenguage: técnico, político, poético, etc. b) Vocabulario: términos clave y distintivos c) Tono: prescriptivo, aseverativo, agresivo, defensivo, etc. d). Estrategias argumentativas: contrastes, analogías, justificaciones cuantitativas, etc. 3. Argumentos (qué se argumenta) a) argumentos centrales del texto b) argumentos principales en relación al tema estudiado c) posicionamiento respecto a los argumentos de otros textos 4. Cosmovisión (desde dónde se argumenta) a) orientación respecto al cambio socio-educativo (funcionalismo vs conflicto)b) perspectiva ontológica (objetivismo vs subjetivismo) c) posicionamiento respecto a cuestiones centrales de la política educativa en general, y en particular al tema de investigación que se lleva a cabo.

\section{¿Cómo se construyen los mapas?}

La construcción del mapa se lleva a cabo a partir de dos ejes cartográficos que representan dimensiones significativas en el campo intertextual, las cuales comienzan a construirse con el relevamiento de los textos tomados para la investigación que se desea llevar a cabo. Estos ejes podrán ser re-diseñados a medida que se avanza con el análisis de los textos y la definición de las perspectivas.

La posición de las perspectivas con respecto a los ejes cartográficos no debe entenderse como ubicación sólo próxima a alguno de los polos de los ejes, sino en el continuo de ambos ejes, horizontal y vertical. La posición en este continuo representa el lugar asignado por los cartógrafos a una determinada perspectiva.

Considerando que no existe una "unidad de 
Figura I. Mapa de Perspectivas indicando flujos discursivos en el debate sobre la posmodernidad en educación comparada y otros discursos relacionados (en PAULSTON, 2001).

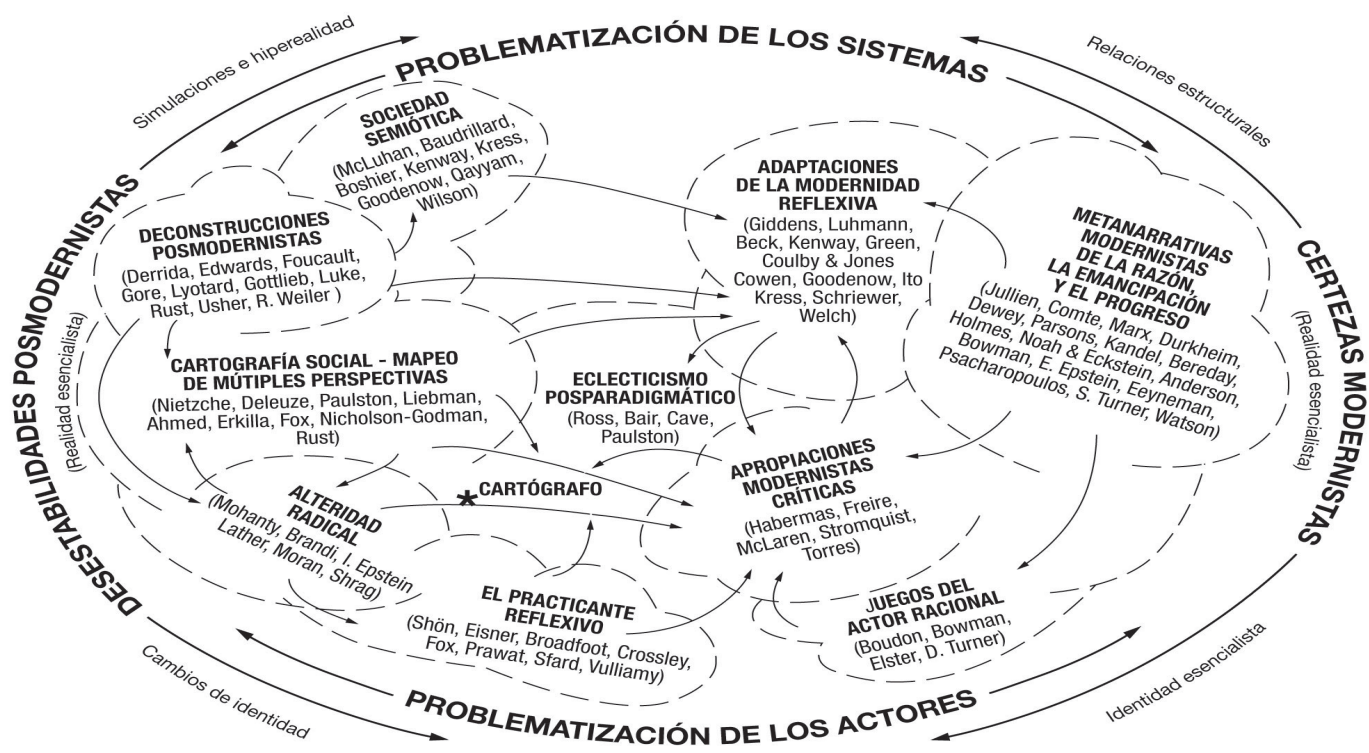

Fonte: PAULSTON, R. G. El espacio de la educación comparada y el debate sobre el posmodernismo. Revista Propuesta Educativa, n. 23,2001 , p. 30

medida" en el método de la cartografía social, dado que se basa en un enfoque cualitativo e interpretativo, la ubicación en el mapa a partir de los ejes cartográficos es sólo a los efectos de la representación. Del mismo modo que el entrelazamiento, contacto o distancia entre perspectiva y perspectiva (obsérvese las Figuras 2, 3, 4 y 5).

Para la identificación y caracterización de las perspectivas, se realiza un proceso iterativo de: 1) lectura de los textos, con especial atención a los argumentos o ideas centrales; 2) identificación provisional, a través de la lectura de los textos, de las principales perspectivas; 3) selección de los textos ilustrativos de cada perspectiva, lo cual puede implicar reducir el número de textos bajo análisis, eliminando aquellos que presentan argumentos ya desarrollados por otros textos; 4) lectura en profundidad de los textos seleccionados enfocada en argumentos, cosmovisiones y características retóricas; 5) revisión de las perspectivas identificadas; 6) incorporación/eliminación de textos ilustrativos (véase GOROSTIAGA, en prensa).

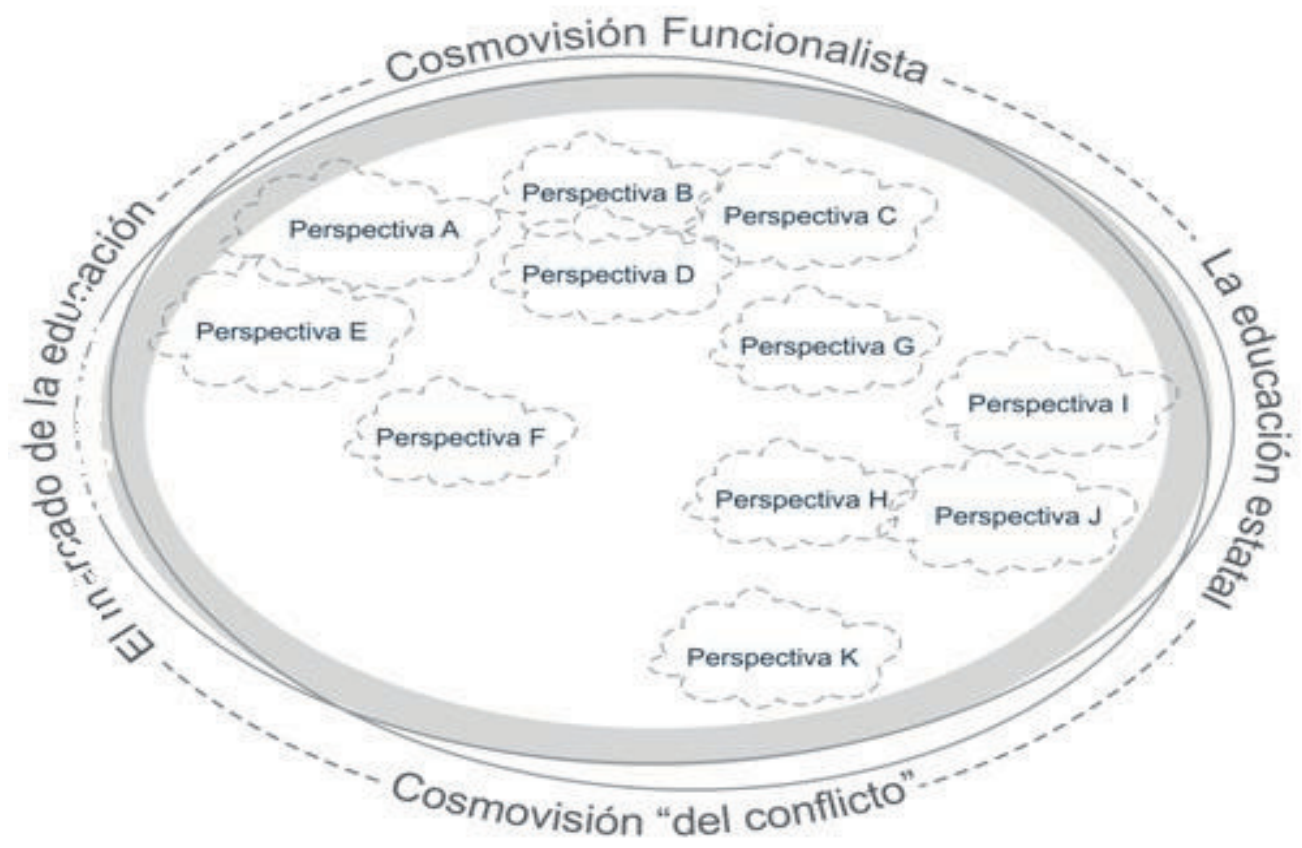

Figura II. Ejes definidos para el "Mapeo de los principales discursos en políticas docentes en Latinoamérica. 1990-2007" (TELLO, 2009) 
En la medida que se vuelve sobre los textos para su relectura y comprensión se realiza una revisión de las perspectivas y su denominación. En este sentido hay que tener en cuenta que el criterio de parsimonia (identificación de un número limitado de perspectivas principales) debe ser balanceado con el criterio de representar el más amplio rango posible de posiciones y voces. Cada texto es comparado con otros textos y asignado a una determinada perspectiva o comunidad, de acuerdo con las dimensiones que surgen como más significativas en el análisis del conjunto de textos - o campo intertextual (PAULSTON, 2001). Esto es, de nuevo, un ejercicio altamente interpretativo y "marcar los límites de las orientaciones textuales o las categorías genéricas es, por supuesto, controversial" (PAULSTON, 1995, p.174).

Dentro de ese campo intertextual, los textos y perspectivas son interpretadas, "clasificadas" y mapeadas topográficamente, esto último con la ayuda de imágenes o representaciones visuales para proveer un "ordenamiento" siempre provisorio. El mapa intenta representar la percepción del investigador/cartógrafo de un determinado fenómeno, percepción que ha sido construida a partir de la exégesis textual. La representación visual también influencia el proceso de identificación de perspectivas ya que, por ejemplo, el mapa puede revelar espacios vacíos que impulsen al cartógrafo a buscar textos y perspectivas localizados en esos espacios.

\section{Las investigaciones utilizando la cartografía social}

Para ilustrar el uso de la cartografía social tomamos tres aplicaciones que hemos desarrollado en nuestras investigaciones:

\section{Investigación sobre la descentralización escolar ${ }^{4}$}

Aquí se analiza cómo se ha entendido y representado la descentralización escolar a nivel global y en el contexto de la Argentina. La descentralización escolar constituye una de las tendencias globales de las políticas educativas de las últimas décadas (WHITTY, POWER, HALPIN, 1998). Estas políticas buscan aumentar la responsabilidad y/o autoridad de las escuelas en diversas áreas, generando considerable controversia sobre sus objetivos y efectos. Alentadas por los organismos internacionales y por los procesos de convergencia

\footnotetext{
${ }^{4}$ Investigación llevada a cabo en el marco de la tesis doctoral de Gorostiaga (2003).
}

de los sistemas educativos, las propuestas de descentralización o "autonomía" escolar se han instalado en los países de América Latina y otras regiones periféricas (GREEN, 1997; MORROW; TORRES, 1995).

La investigación incluyó la identificación y caracterización de las principales perspectivas sobre la descentralización escolar - y de sus interrelaciones -, a través del análisis interpretativo de textos académicos e institucionales, referidos a casos nacionales o con un enfoque internacional ${ }^{5}$. En la exégesis textual se prestó particular atención a lo que Fairclough (1992) Ilama la "intertextualidad manifiesta" en forma de referencias y citas de otros textos para mostrar los distintos tipos de relaciones existentes entre las perspectivas. Al mismo tiempo se utilizaron mapas bi-dimensionales, los cuales contribuyen a la representación de la descentralización escolar como un debate intertextual entre perspectivas múltiples e inter-relacionadas que argumentan acerca de los beneficios y los perjuicios de diferentes políticas y propuestas.

En el caso del debate global (véase la Figura III), se identificaron siete perspectivas. Tres de ellas (Sistemas Eficientes, Mercados Educativos y Fortalecimiento Local) proponen en forma entusiasta distintos mecanismos de descentralización escolar. Hay una cuarta (Balances Pragmáticos) que apoya la descentralización pero con ciertas reservas, una quinta (Participación Democrática) propone una alternativa democratizadora de descentralización, y otras dos (Crítica Radical y Crítica de la Efectividad) se oponen abiertamente en base a distintos argumentos. En este mapa se incluyen textos que ilustran posibles combinaciones entre más de una perspectiva y las flechas están usadas para indicar relaciones de oposición o de adopción de argumentos entre las distintas perspectivas. En este caso, la CS permite ir más allá de la oposición centralización-descentralización para identificar perspectivas que, por ejemplo, ligan la opción por una política de mayor autonomía escolar a distintas cosmovisiones y objetivos políticos (que priorizan la eficiencia, la libertad individual o la democratización social) o que se oponen a ella basadas en diferentes argumentos (de igualdad y democratización versus efectividad académica).

Como se puede observar en el mapa no siempre se utilizan Comunidades Discursivas en el sentido de Tello (2009), dado que depende del investigador si considera apropiado el

\footnotetext{
${ }^{5}$ Se analizaron unos 100 textos para el nivel global y 28 para el caso argentino, de los cuales se seleccionaron 29 y 14, respectivamente, para ilustrar las perspectivas identificadas. Para el nivel global se utilizaron principalmente textos producidos en el mundo anglosajón (por su impacto en el discurso educativo supranacional) y de difusión internacional.
} 


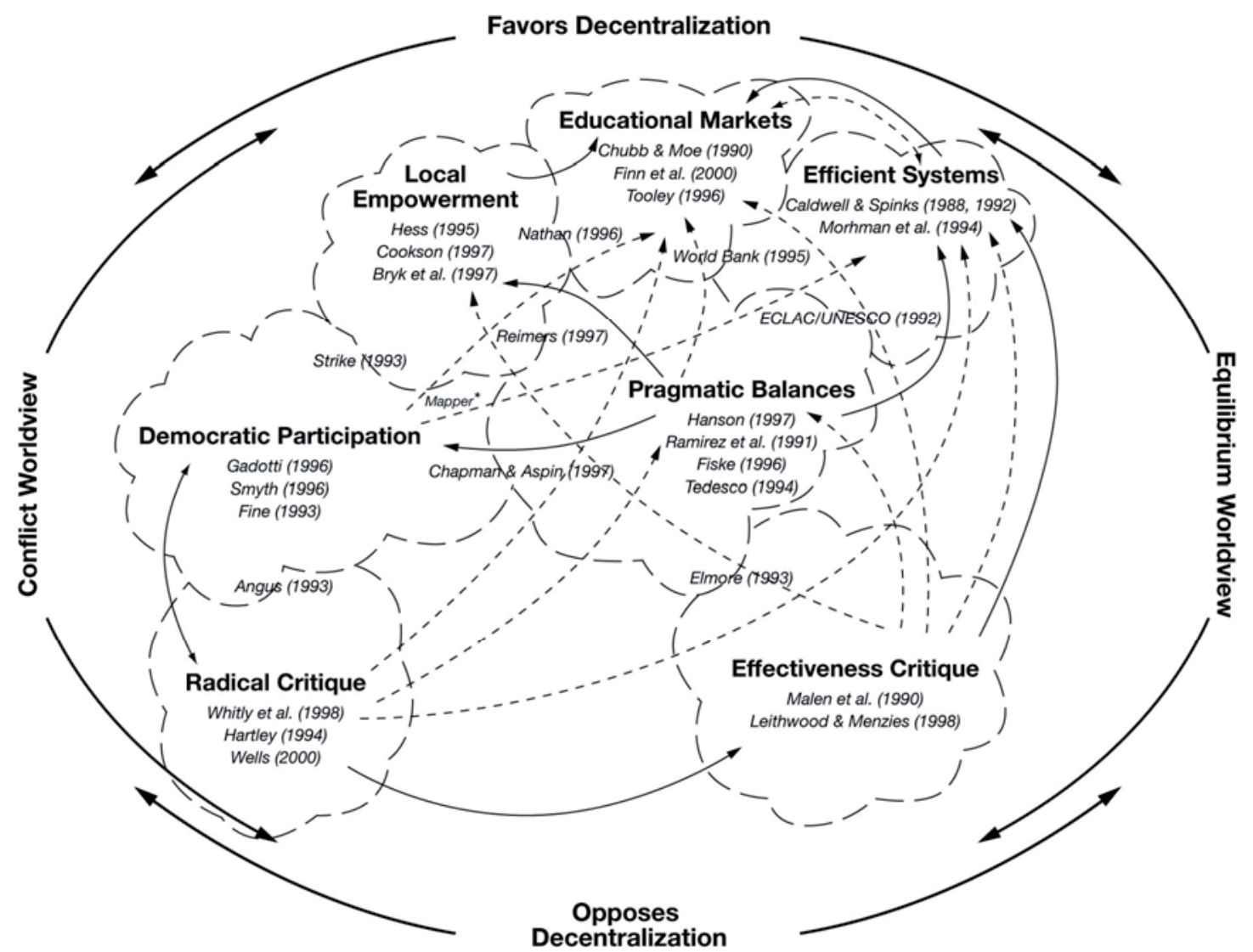

Figura III. Mapa del debate global sobre la descentralización escolar como campo intertextual (en GOROSTIAGA, 2003; GOROSTIAGA, PAULSTON, 2004).

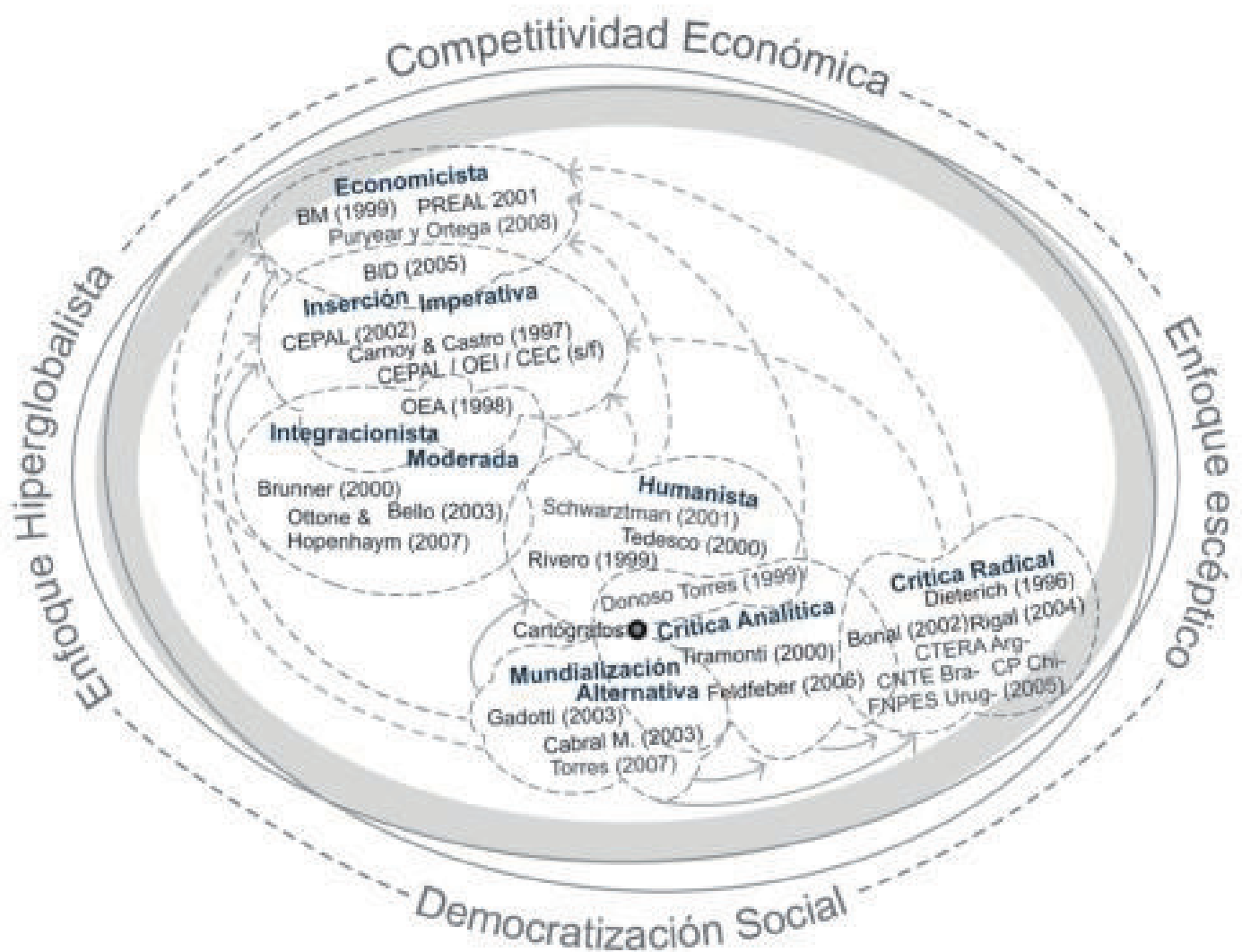

Figura IV. Mapa del debate sobre globalización y reforma educativa en América Latina como campo intertextual (GOROSTIAGA, TELLO, MARTINÉ, 2008) 
agrupamiento en comunidades o desarrollar un mapa sólo con Perspectivas

\section{Investigación Globalización y educación en América Latina ${ }^{6}$}

Esta investigación llevó por título "Un mapeo del discurso sobre globalización y educación en América Latina" teniendo en cuenta que el impacto y direccionalidad de los procesos de globalización en relación con la educación en América Latina han sido conceptualizados de diversas maneras por distintos actores, constituyéndose el discurso sobre esta cuestión en un espacio de disputa de significados con implicancias para la práctica y la política educativa. Entre otros aspectos, se han verificado debates sobre la convergencia de los sistemas educativos nacionales, la adaptación de la educación a las demandas de la economía y de la "sociedad del conocimiento", el desarrollo de iniciativas y empresas educativas transnacionales, y el predominio de una ideología neoliberal modernizante a nivel global. Se analizaron unos 50 textos, de los cuales fueron seleccionados 24 para el mapeo, incluyendo documentos producidos por organizaciones multilaterales y no gubernamentales, así como textos académicos que discuten las implicancias de la globalización en el campo educativo latinoamericano, con el objetivo de caracterizar críticamente y comparar las principales perspectivas sobre el tema.

El mapa fue construido en base a dos dimensiones. La dimensión horizontal se relaciona con la visión de los procesos de globalización, con el enfoque hiperglobalista en un polo y el enfoque escéptico en el otro; de acuerdo con la tipología presentada en Tikly (2001), el centro de esta dimensión estaría asociado al enfoque transformacionalista. La dimensión vertical, por su parte, alude a valorizaciones sobre las reformas educativas y sus fines, con la competitividad económica en un polo y la democratización social en el polo opuesto.

\section{Investigación sobre políticas docentes en Latinoamérica $^{7}$}

El objetivo de la investigación consistió en realizar un mapeo de los principales discursos sobre políticas docentes en Latinoamérica durante el período 1990 - 2007, que tenía una pregunta inicial

\footnotetext{
${ }^{6}$ Esta investigación se llevó a cabo en la Escuela de Posgrado de la Universidad Nacional de San Martín. Director: Jorge Gorostiaga. Asistentes de Investigación: César Tello y Eduardo Martiné.

${ }^{7}$ Dicha investigación se llevó a cabo en el marco de la tesis de maestría en políticas y administración de la educación de la UNTREF de César Tello (2009) "Un mapeo de los principales discursos en políticas docente en América Latina (1990-2007)".
}

con sus respectivos objetivos: ¿Cuál sería uno de los mapas de los principales discursos sobre políticas docentes en Latinoamérica durante el período 1990 - 2007? y como objetivos se plantearon: a) identificar y describir los principales discursos sobre las políticas docentes en Latinoamérica durante el período 1990 - 2007 y b) analizar y mapear los principales discursos sobre las políticas docentes y sus interrelaciones en Latinoamérica durante el período 1990-2007

En la pregunta de investigación de algún modo se define el enfoque que se asume desde la CS: "uno de los mapas posibles", esto es, el que el investigador/cartógrafo va construir. Otro investigador, inclusive, analizando los mismos textos puede obtener o construir otro mapa. En esta investigación existen dos tipos de interconexión argumentativa, en primer lugar lo que se denominado "comunidades discursivas" que comparte una visión acerca de las políticas en Latinoamérica y dentro de cada comunidad discursiva se observan declinaciones: las perspectivas. En esta investigación se analizaron 57 textos seleccionados dentro de un universo de 127 textos.

\section{Notas Finales}

Una de las cuestiones centrales de la cartografía social es que permite visualizar -y eventualmente integrar- diferentes perspectivas que conceptualizan el fenómeno, y cómo estas interactúan o se interrelacionan. Por otra parte, aparece como un intento válido de responder al desafío de la post-modernidad de dar cuenta de diferentes perspectivas del conocimiento asumiendo una posición frente a la problemática (en los mapas véase posición del cartógrafo) pero tratando de interpretar y reflejar esas diferentes perspectivas en sus propios términos.

En la medida en que se busque comprender la lógica y las implicancias de la multiplicidad de discursos o perspectivas discursivas, así como su interrelación, la CS se constituye como una herramienta de análisis sumamente valiosa que puede ser combinada con otros métodos y técnicas para el estudio crítico de diversos problemas educativos. Además la CS ofrece a los investigadores una manera - entre otras posibles - de incorporar representaciones visuales al análisis y presentación de hallazgos, un tipo de representación que es poco usado y potencialmente valioso en el campo educativo y que constituye en sí mismo un tipo particular de discurso (RUITENBERG, 2007).

En este artículo hemos explicado los principales rasgos del enfoque de la CS y la metodología de mapeo. Además, hemos mostrado tres ejemplos de la aplicación de la CS al mapeo de debates sobre 


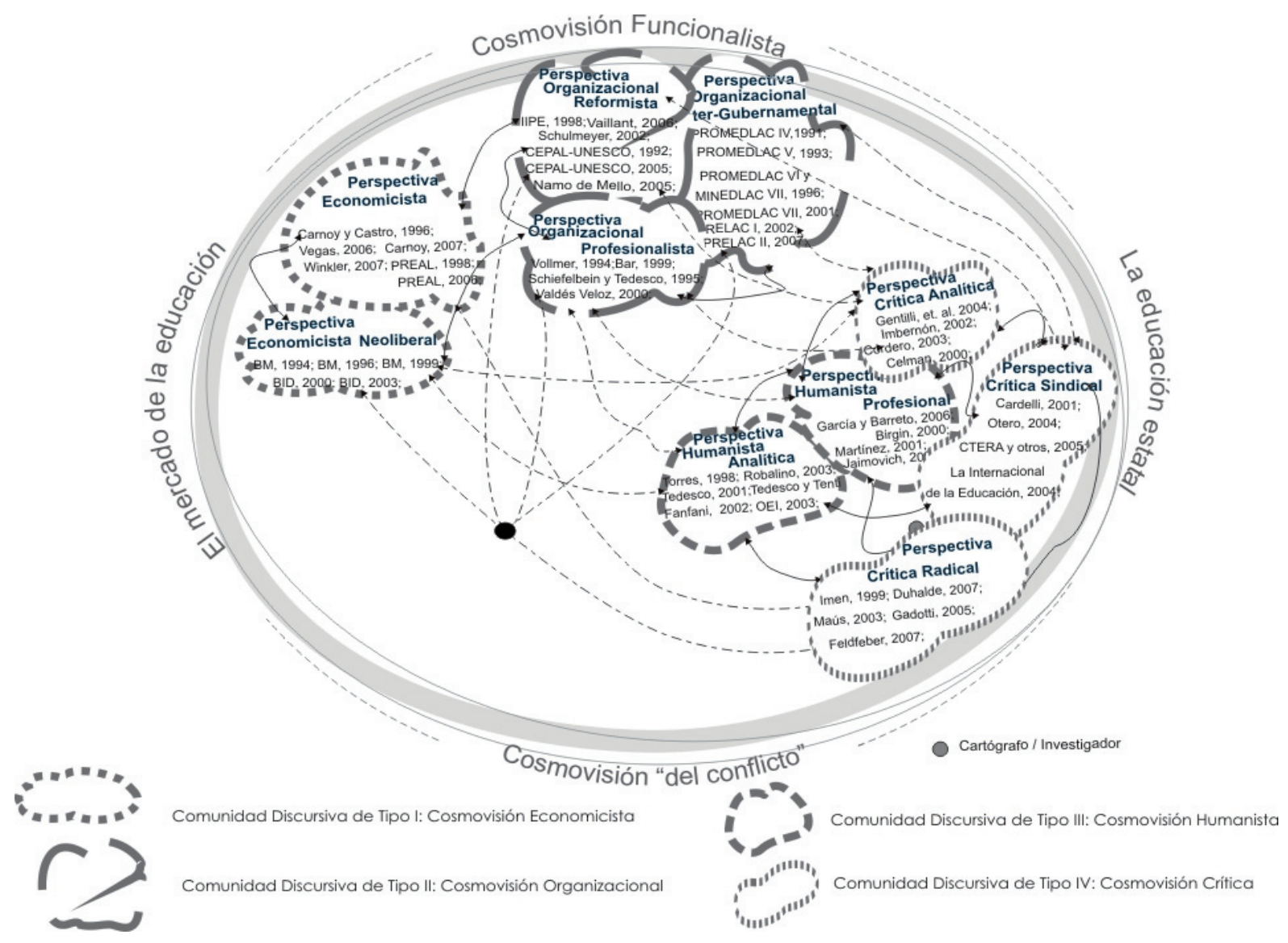

Figura V. Los principales discursos en el debate de políticas docentes en Latinoamérica. 1990-2007 (TELLO, 2009)

temas de política educativa. A través de la aplicación del mapeo del debate, lo que se busca lograr es una mejor comprensión del tema, presentando los argumentos y contra-argumentos de distintas perspectivas que en un análisis más general son pasados por alto o permanecen en una zona oscura y que implican distintas opciones de política. Esto no implica ignorar que toda política es moldeada también por otros debates y discursos relacionados así como por las relaciones de poder existentes que resultan en particulares usos o no-usos de argumentos. La idea de debate ayuda a iluminar las tensiones y coincidencias entre distintas formas de ver y a interpretar el discurso como un espacio en el que múltiples perspectivas entran en dialogo y confrontación, "como un sitio y objeto de lucha donde diferentes grupos buscan la hegemonía en la producción de significado e ideología" (PAULSTON, 1995, p. 162).

Además del valor heurístico de este tipo de análisis, puede haber un uso más político para aquellos que en ámbitos nacionales o locales promueven o critican determinadas políticas o programas de reforma educativa. Desde la perspectiva de los decisores puede argumentarse que para la elección entre opciones de política, tan importante como los datos cuantitativos es entender los diferentes argumentos que configuran esas opciones. Por otra parte, desde el punto de vista de los países latinoamericanos, además de identificar y criticar el discurso educativo dominante, puede resultar importante analizar el debate de ideas a nivel global incluyendo aquellas perspectivas que - siendo menos poderosas en términos de qué actores las respaldan y difunden - tienen un potencial anti-hegemónico y transformador.

\section{Referencias}

ANDRADE, H.; SANTAMARIA, G. Cartografía social para la planeación participativa. In: ANDRADE, H.; SANTAMARIA, G. Memorias del curso: participación comunitaria y medio ambiente. Proyecto Capacitación para profesiones del Sector Ambiental. Santa Fé de Bogotá: Ministerio del Medio Ambiente de Colombia e ICFES, 1997. p. 3-16.

BUZAI, G. D. Mapas sociales urbanos. Buenos Aires: Editorial Lugar, 2003.

CLAVAL, P. La geografía cultural. Buenos Aires: Eudeba, 1999. 
FAIRCLOUGH, N. Discourse and social change. Cambridge, UK: Polity Press, 1992.

FOLLARI, R. Teorías débiles: para una crítica de la deconstrucción y de los estudios culturales. Rosario: Homo Sapiens, 2002.

FOUCAULT, M. Of other spaces. Diacritics, v. 16, n. 1, p. 22-27, 1986.

GIDDENS, A. Central problems in social theory: action, structure and contradiction in social analysis. Berkeley: University of California Press, 1979.

GOROSTIAGA, J. Mapping perspectives on school decentralization: the global debate and the case of Argentina. 2003. 198 p. Tesis (Doctorado en Análisis Social y Comparado en Educación) - University of Pittsburgh, Pittsburgh, 2003

GOROSTIAGA, J. Esquema de análisis para los textos en la cartografía social. (mimeo), 2007.

GOROSTIAGA, J. La cartografía social: aportes al análisis del discurso en educación. In: PINI, M. (comp.). Discursos y educación: herramientas para un análisis de los discursos en educación. Buenos Aires: UNSAM Edita. (En prensa).

GOROSTIAGA, J. M.; PAULSTON, R. G. Mapping perspectives on school decentralization: the global debate and the case of Argentina. In: NINNES, P.; METHA, S. (comps.). Re-imagining comparative education: postfoundational ideas and applications for critical times. London: RoutledgeFalmer, 2004. p. 255-284.

GOROSTIAGA, J; TELLO, C.; MARTINÉ, E. Un mapeo del discurso sobre globalización y reforma educativa en América Latina. In: V Jornadas de Sociología de la UNLP, La Plata, diciembre, 2008.

GREEN, A. Education, globalization and the nation state. London: Macmillan Press, 1997.

HABEGGER, S.; MANCILA, I. La cartografía social en las prácticas contrahegemónicas o la cartogafía social como estrategia para diagnosticar nuestro territorio. 2006. Disponible en: <http://areaciega.net/index.php/ plain/>. Acceso: 2 jul. 2009.

LIEBMAN, M.; PAULSTON, R. Social cartography: a new methodology for comparative studies. Compare, v. 24, n. 3, p. 233-245, May, 1994.

MARTON, F. Phenomenography. In: HUSEN, T.; POSTLEHWAITE, T. N. (comps.). The International Encyclopedia of Education. 2. ed. Oxford: Pergamon Press, 1994. v. 8, p. 4424-4429.

MORÍN, E. La cabeza bien puesta. Argentina: Nueva Visión, 1999.

MORROW, R. A.; TORRES, C. A. Social theory and education: a critique of theories of social and cultural reproduction. Albany, NY: SUNY Press, 1995.

NINNES, P.; METHA, S. A meander thorugh the maze: comparative education and postfoundational studies. In: NINNES P.; METHA, S. (comps.). Re-imagining comparative education: postfoundational ideas and applications for critical times. London: RoutledgeFalmer, 2004. p. $1-18$.
PAULSTON, R. G. Mapping knowledge perspectives in studies of educational change. In: COOKSON JR., P. W.; SCHNEIDER, B. (Eds.). Transforming schools. New York: Garland, 1995. p. 137-179.

PAULSTON, R. G. Preface: four principles for a non-innocent social cartography. In: PAULSTON, R. (comp.). Social cartography: mapping ways of seeing social and educational change. New York: Garland, 1996. p. 15-24.

PAULSTON, R. G.; LIEBMAN, M. Social cartography: a new metaphor/tool for comparative studies. In: PAULSTON, R. G. (Ed.). Social cartography: mapping ways of seeing social and educational change. New York: Garland, 1996. p. 7-28.

PAULSTON, R. G. El espacio de la educación comparada y el debate sobre el posmodernismo. Propuesta Educativa, Buenos Aires, v. 10, n. 23, p. 18-31, Dic. 2001. (Versión en portugués: PAULSTON, R. G. Mapeando a educação comparada depois da pós-modernidade. Educação, Sociedade \& Culturas, Porto, n. 16, p. 203-239, 2001.)

PETERS, M.Qué es el postestructuralisrno?. Cuadernos de Pedagogía, Rosario, Laborde Editor, v. 4, n. 8, p. 39-66, abr. 2001.

RUITENBERG, C. Here be dragons: exploring cartography in educational theory and research. Complicity: an International Journal of Complexity and Education, v. 4, n. 1, p. 7-24, jul. 2007.

SILVA, T. T. da. Espacios de identidad: nuevas visiones sobre el currículum. Barcelona: Octaedro, 2001.

TELLO, C. Un mapeo de los discursos de las políticas docentes en Latinoamérica 1990-2007. 2009. 307 p. Tesis (Maestria) - Programa de Posgrados en Políticas y Administración de la Educación, Universidad Nacional de Tres de Febrero, Buenos Aires, 2009.

TIKLY, L. Globalisation and education in the postcolonial world. Comparative Education, v. 37, n. 2, p. 151-171, May, 2001.

VÁSQUEZ ROCCA, A. El Hipertexto y la lógica del laberinto: textualidad, redes y discurso ex-céntrico. Revista Latinoamericana de Filosofía Aplicada, Instituto de Filosofía, Pontificia Universidad Católica de Valparaíso, 2001. Disponible en: <http://www.geocities.com/filosofia_ aplicada/HIPERTEXTO.htm>. Acceso: 2 jul. 2009.

WALLERSTEIN, I. Las incertidumbres del saber. Barcelona: Gedisa, 1997.

WHITTY, G.; POWER, S.; HALPIN, D. Devolution and choice in education: the school, the state and the market. Buckingham: Open University Press, 1998.

Recebido em 08/07/2009

Aceito em 12/08/2009 